was engorged, and its tinge was imparted to it and the adjoining structures. The large intestines were clogged with this viscid, glutinous secretion, which hitherto had marked the excretions, and they were replete with excrementitious matter; on cleansing their interior, there was no congestive or ulcerative sign whatever. A vivid redness, but no ulceration, was apparent in the fauces, and the tonsils were increased a little in size. The remaining viscera call for no further comment.

Post-mortems, however, throw but little light on these malignant diseases, notwithstanding a minute investigation into the appearances of the organs furnished by the fatal ending of the above case, there was no proof of organic lesion, but more of what has been termed the lesion of functionthis depending upon the vitiated condition to which the organs have become subservient from the mephitic agent (supposing it to have been the cause) floating in the blood.

The treatment which such cases require must be obvious, their most prominent features must be lessened by counteracting remedies, and the great state of depression invariably attendant in all malignancies, meet with agents calculated to check the advancement of the disease, and restore, by their tonicity, the disordered functions and diseased parts to health. In all stich cases, the milder the remedies, the more beneficial will be the result; provided the inherent power of the system be not overcome, the effects of the morbific agent will be somewhat retarded, and the disease will thereby be prevented from assuming a more serious or malignant form; for when we consider the train of evils that may ensue from any of the malignant exanthems, it behoves us not to disregard any of the measures because they are mild, and offered in opposition to a vigorous disease, when we know that they are best calculated to afford relief, and subsequently, it is to be hoped, a cure. Variola more often than scarlatina, and this latter more frequently than rubeola, are the subjects of malignancy, and the virulence and danger of such maladies range accordingly proportionately. But then it is the nature of these diseases to be thus proportionally severe, and successively to become affected, and more the subjects of malignancy. When. ever they become so, they always earlier or later assume the typhoid type, and this not unfrequently at an early stage of the disease. A generous diet, with free ventilation and soothing remedies, are the means most calculated to ensure a cure. The chlorate of potash, so vaunted a remedy in these affections, does not merit the qualifications bestowed upon it by some; in many instances it has appeared inert, and I have found equal benefit, and oftentimes more, in some cases, from other remedies of less reputed worth. That such diseases should require more than a passing glance, the frequent occurrences of fatal terminations clearly prove.

Church-street, Shoreditch, May, 1849.

\section{ON THE TREATMENT OF SOME CUTANEOUS DISEASES.}

To the Editor of The LANCET.

Sir,-In a review of Mr. Hunt's work on "Diseases of the Skin," which recently appeared in THE LANCET, you expressed a hope that other members of the profession would take up the subject, and test, by experience, the soundness of the views which were therein advanced: I therefore forward a selection of cases which have been treated on the principles proposed by Mr. Hunt.--I am, Sir, your obedient servant,

SaMuel Grifrith,

Late House-Physician to King's College Psoriasis.

R. B - aged nine, is a native of Bow, where he has usually resided. His general health has always been good. About twelve months since, in falling, he abraded the skin of the left knee; and from the constant irritation of his trousers, the wound became a large, thongh superficial sore. Two months after this he observed white scales form over the not quite healed wound; and at the same time an eruption of like character appeared on the right knee, elbows, forearms, and trunk. He has for some time been using ointment with, as he thinks, slight benefit.

Dec. 31st, 1846. At present he is a fine, healthy-looking lad, with a florid complexion. There are several large, irregular patches on the knees and elbows, and smaller ones scattered over every part of the body, excepting the face; they are covered with numerous shining, silvery scales; the edges are raised and irregular; there is no itching or uneasi- ness; bowels free; tongue clean. Ordered, nitro-muriatic acid, ten minims; infusion of cinchona bark, a pint. Mix. An ounce to be taken three times a day.

He continued taking this medicine up to the 20th of January, when there was an improvement in some of the patches; a few of the smaller ones had healed. He was directed to continue this medicine, and apply the nitrate of mercury ointment.

Feb. 10th, 1847.- Many of the patches have disappeared, only a slight brown stain; the others are smaller, and less scaly. Repeat the remedies.

26th.-The eruption, which had nearly passed away, is now returning in many places. From this period, up to May 29 th, various external applications were used, as the nitrate of silver, tincture of iodine, \&c. Liquor potassæ, and a compound of arsenic-with-mercury, (not to salivation,) were also given internally, but without benefit, for any temporary amendment that might have taken place was soon followed by an increase of the disease.

May 29th.-At present there are numerous small patches about the trunk; others on the arms, spreading, as it were, from the elbow-joints on either surface; the largest are scattered on the anterior part of the knees and legs; the patches are much raised, scaly, and itchy, which induces the boy to scratch, when an ichorous fluid is discharged, forming a hard cake over several of them. The other functions appear healthy. Ordered, Fowler's solution of arsenic, a drachm; distilled water, seven drachms. Mix. Forty minims to be taken, in water, thrice daily.

June 19th.-A marked change in the character of the eruption has taken place; it is paler, less scaly, and there is a total absence of the irritation which so much annoyed him a fortnight since. No fresh patches have appeared, nor are there any unfavourable symptoms from the use of the arsenic; the only indications of the system being under its influence are the amendment of the disordor, and an almost imperceptible inflammation of the conjunctiva.

He continued the same treatment up to July 15 th, when all trace of the disease, with the exception of a slight discoloration of the skin, had disappeared.

Feb. 2nd, 1848. - No return of the eruption; the skin is quite free from spots or roughness.

March 30th.-Two small spots having shown themselves"on the right knee, he recommenced taking the drops a fortnight since. The spots are now almost gone. To continue the arsenic, in four-minim doses, for a month longer.

Feb. 1849. - The boy is well, and entirely free from his former complaint, of which he has had no return.

\section{Lepra.}

James P_, aged twelve, is a stout, healthy-looking. boy, somewhat predisposed to scrofula, but has generally enjoyed excellent health; his parents and brothers are free from eruptive disorder. About two months before consulting me, he perceived an eruption, which, commencing at the elbows, soon spread over the backs of both arms. Occasional aperients, and other medicines, were exhibited, but the disease continued spreading. It first appeared as small clusters of papulæ, each of which became covered with a scale; these subsiding, others sprang up around them, and formed the circular, scaly patches, with elevated borders, characteristic of lepra vulgaris. Many of these have now become confluent, presenting the ordinary appearance of psoriasis; while others still retain the circular form of lepra. The eruption extends over both arms, neck, and trunk.

Dec. 28 th, 1846.- There being nothing to contraindicate its use, Fowler's solution of arsenic was given, in doses of five minims, with his meals, three times a day.

Jan. 6 th, 1847.-The psoriasis continues about the same, but the body is nearly covered with a papular eruption, (lichen sifnplex.) The itching is troublesome, especially in the night, preventing sleep. The conjunctiva is affected. Continue the arenic.

16th.-The lichen has died away, leaving the skin coated with fine scurf. The lepra is fading, no fresh patches have appeared; the sub-maxillary glands are swollen and tender, and he complains of weakness of the eyes. The conjunctiva being more injected than is consistent with the proper action of the medicine, the dose was reduced to four minims.

30th.-The eruption is fast fading; the swelling of the glands has subsided, but the conjunctiva remains affected. The general health continues very good. Skin free from the lichenous scurf.

Feb. 15.-The lepra is quite gone, but has left the usual brown stains. The arsenic was continued to the end of March. 
when the skin had resumed its natural appearance. There had been no return of the eruption when $I$ saw the patient four months subsequently.

Psoriasis Inveterata.

Mrs. W- aged forty-seven, a native of Bristol, but has generally lived in London; is of healthy family, but her four brothers are subject to psoriasis, appearing or exacerbated at the spring and fall of the year. Previous to her marriage she was very healthy, but in the year 1827 , having been confined with twins, both of which she suckled, avoiding beer, and living lower than she ought to have done, her health began to fail, and at the end of nine months a scaly eruption appeared on the backs of the hands, which in a few weeks had spread over the arms. She was attended by one of our most celebrated hospital surgeons, who told her to give up suckling, and, according to her account, stated, that all her blood being diseased, it would be necessary to remove it; she would then get well. To this end he bled her freely, applied leeches to the temples, and directed her to live entirely on bread-and-milk. Under this treatment she became delirious, and within fourteen days the eruption had spread nearly over the whole surface of the body, excepting the face, which has always been entirely free.

In the course of three months, when her health had somewhat improved, notwithstanding occasional bleedings, but without any amendment in the eruption, Mrs. W- went into the country, where her medical attendant advised good diet, small doses of elixir of vitriol, to drink freely of Cleider tea, and apply some white ointment to the eruption. As she gained strength under this treatment the eruption died away. She was confined again in the following year, and has subsequently had seven children; after each confinement a similar eruption appeared, and more rapidly on each successive occasion. Outward applications and other means were resorted to, but the disease appeared to be most influenced by weaning the children, which she was compelled to do, for though there was a free secretion of milk, it was poor and watery, and the children refused it, preferring food.

Dec. 16th, 1847.- Her last child was born four years ago, since which period the eruption has continued worse in spring and autumn, up to the present time. It is now more extensive than it has before been, the upper and lower extremities being nearly covered, and there are many large patches on the trunk. The affected part is raised, with large white scales, of a dusky red colour, very irritable, and in some parts fissured; she has varicose veins, and several small sores on the legs, brought on by scratching, which she is unable to avoid. Her health is indifferent, though as good as it has been for the last four or five years. The catamenia have ceased for three years. Bowels sluggish; appetite bad; frequent headach; tongue foul. The state of the digestive organs having been rectified by small doses of blue pill and colocynth, five minims of Fowler's solution were given with the food three times a day; the sores dressed with spermaceti cerate and bandaged; to have plain but nutritious diet.

Jan. 14th, 1848.-The eruption is already presenting signs of amendment, being less irritable and scaly; the conjunctiva is affected, and she has had a slight attack of gastritis, to which she has always been subject on the disappearance of the eruption, though never when it was fully out. Thinks herself stronger; appetite indifferent; tongue clean; bowels regular. Continue the arsenic.

Feb. 12th.-Since the last report, there has been a much more marked improvement. The eruption generally is fading, and does not cause so much annoyance; there are no fresh patches; the varicose veins on the legs are better; she has more appetite; no return of gastritis; conjunctiva is stil injected, but not sufficiently so to cause annoyance.

March 15th. - She feels herself much better; the scaliness and prominence of the disease has left in many places; she can now rest comfortably, as she feels no inclination to scratch conjunctiva still affected; she has no gastritis; the tongue is clean; bowels regular; appetite good.

April 16th.-The whole diseased surface is free from scaliness; the ulcers on the legs have been for some time healed; the varicose veins are less distended; and she can wear her shoes, which she had been unable to do for some time previously. Repeat the medicine.

June 14th.-Health appears completely restored; the only trace of eruption is the brown state of the skin, which, otherwise, seems to be quite sound.

The arsenic was continued, in four minim doses, for three months longer, when the discoloration of the skin had disappeared.

December.-No reappearance of the disease.
Psoriasis.

J. R- ared twenty-seven, came to consult me about an exuption which first appeared when he was four years old otherwise he has enjoyed good health. The disease, which has never entirely left him, notwithstanding the frequent and prolonged use of medicine, becomes more extensive and irritable every spring, but fades as winter advances; four months since, it was worse than ever; he has been taking medicine and using external applications, but without any benefit.

May 17th.-An eruption presenting the ordinary characters of psoriasis inveterata, covers the extensor surface of both hands, arms, and legs; there are also a few small patches on the trunk and back of the legs; skin is much thickened, scaly, irritable, redder than usual, and fissured; has slight feverishness; tongue being furred; skin dry and hot; pulse 100, full. These symptoms having been removed by use of purgatives and small doses of tartar-emetic, the arsenic, in five minim doses, was commenced May 22nd.

June 8th.-The disease is fading, and but slightly annoys him; two or three new places have appeared on the trunk conjunctiva is slightly affected, but there is no irritation of the stomach. Repeat the arsenic.

28th.-Improved in every respect; no fresh spots.

July 16th.-He has neglected taking his medicine for some days past; the scales are thicker; and some new patches have formed. The redness of conjunctiva has gone. Resume arsenic.

Sept. 16th.-The complaint has entirely left him. The arsenic was again intermitted for a short time at the end of July, with the same unfavourable effect as previously. Continue the medicine for three weeks.

Feb., 1849.-No return of the disease.

Stephen F- aged forty, is of temperate habits; leads a sedentary life, but with the exception of slight ailments, and the complaint for which he applied to me, has always had excellent health. His mother was subject to a scaly exuption on the elbows, no other member of his family. He states that it is now twenty years since the disease first manifested itself on his right arm; it was then about the size of a shilling, and though not irritable, he was unable to resist the inclination to scratch it; to this he attributes its subsequent spread. In the course of a few months the other arm became studded with numerous small patches. During this long period, he has used and persevered in a great variety of remedies, bot without permanent benefit; he has twice taken arsenic under the direction of his medical adviser, but was unable to continue it long, as it caused very severe gastric irritation, and the disease seemed to extend and become more irritable under its use; he therefore requested me not to prescribe anything of this kind. When not taking medicine, the disease is much influenced by the season of the year as well as by his mode of living, any irregularity causing an exacerbation. Has not been under medical treatment for some time past.

At present is a stout, healthy-looking man, is married, and has several children; he complains greatly of the intense itching and pain in the eruption, which exists on the flexure side of both elbow-joints, and bleeds on extending the arms which, on account of this, he cannot fully do. There being some heat and dryness of skin, with furred tongue and constipation, a dose or two of calomel and colocynth, with antiphlogistic regimen, was ordered for a few days; these symptoms having subsided, he commenced the Fowler's solution August 16th, 1847. When the disease had come under the arsenical influence, as indicated by the state of the conjunctiva, the disease began to amend, and in four months had entirely left him. Being tired of the medicine, and, moreover thinking himself thoroughly recovered, he discontinued its use. In two or three weeks, perceiving symptoms of the reappearance of his disorder in the same locality, he was induced to persevere in his medicine for three months after the eruption had left him. It has not since appeared. The arsenic, on this occasion, did not irritate any part of the gastric system.

I cannot forbear offering a few observations on some of the principal points in the cases above related, especially with regard to the mode of administering arsenic. This medicine, prescribed by others, has frequently been productive of great benefit in the cure of chronic skin diseases, but no author is to be found who has met with such marked success in its use as Mr. Hunt. At its first introduction, as indeed often happens with other agents, its influence was decided in the hands of many. Not-so in later cases; and many practitioners have, by degrees, come to lose all faith in it. This may, I think, be readily accounted for. A man unaccustomed to arsenic would be very cautious in prescribing it, and this very fear would 
lead him to use it in something like the manner in which its beneficial effects are most apparent. As, however, his confidence in it grows stronger, and his fear of its baneful effects subside, he is induced to give it in larger doses, hoping more quickly to eradicate the disease; this not only interferes with its proper action, but also causes it injuriously to affect the system.

Arsenic should never be given when there is any inflammatory action about the system, otherwise this state is sure to be increased; all the functions must first be brought into a healthy condition.

Whatever be its modus operandi in the cure of skin disease, my experience goes decidedly to establish the tonic action of arsenic. Under its use alone, and without any alteration in diet or residence, I have seen marlied improvement, not only in the disease, but in the general health of the patient; these, in fact, seem usually to go hand in liand. Herein is shown its specific action, as distinguished from other tonics; these, when exhibited, cause improvement of the health, but exert no apparent influence on the eruption. I am aware that in this opinion I differ from one of our chief authorities, Dr. Pereira, who, though he has tried it extensively, and with his attention directed to this point, has been unable to satisfy himself of its right to be classed as a tonic; the appetite of his patients sometimes improved while taking it, in no other respect was its tonic action shown. Vogl, (Pharmakodyn,) and many others, have arrived at the opposite result.

And here I would advert to the common mode of giving arsenic-i.e., in increasing doses. What tonic could be given in such a manner without danger of producing severe constitutional disorder? It is the peculiarity of this class of medicines, that their action is so gradual as to be almost imperceptible, days, or perhaps weeks, being required to show any decided effect. Thus is it with arsenic; though its action is more manifest from the complaint being external. Arsenic ought, therefore, to be given in moderate, or what might be called tonic doses, at first, until the system comes under its influence; these must be diminished, if it affects the patient too severely. The action of arsenic seems to be more direct on the tegumentary tissues, the skin, and mucons membranes, and its influence is nearly always first manifested on the tarsal conjunctiva; hence we have a ready guide to its exhibition. The degree at which we wish to arrive, is a very slight injection of this membrane; if it goes beyond that point, and more severe irritation is caused, then the eruption becomes inflamed, and even spreads; this may in almost every case be prevented by diminishing the dose. If the system be kept in this state, no injurious operation need be feared. Five minim doses of Fowler's solution may be safely given at first to a person who is moderately strong, has not had his constitution injured by its abuse, and who is not unusually intolerant of it. Cases will, however, present themselves to the practitioner, in which it will be advisable to begin with a smaller dose than this; indeed, if there be any doubt, it is better to give the smaller dose, two, three, or four minims, and pursue it for a longer time; the system is sure, though at a later period, to come under its power. The disease is generally seen to date its improvement from the time of the first affection of the conjunctiva.

Again, arsenic should be taken with the meals, or on a full stomach; this serves to dilute it, and prevents the gastric irritation which so frequently follows when it is exhibited without this precaution.

The decided infuence which this remedy has over certain eruptive disorders, is shown, not merely by their amelioration under its proper use, but more manifestly by omitting it for a time, as I have frequently done, and invariably has the disease recommenced spreading as soon as the system had got rid of the arsenic, as shown by the subsidence of the conjunctivitis, and its course has been again stayed when the medicine has been resumed sufficiently long.

The rule which Mr. Hunt has laid down in his very excellent work-viz., " the arsenical course should be protracted (in reduced doses) as many months after the final disappearance of the disease, as it had existed years before."* Though perhaps not necessary in every case, it is always advisable, as at this time it is much less difficult to eradicate the tendency to the disease, than, on its re-appearance, to travel again over the same ground.

In conclusion I would observe, that though, as is well known, injurious effects have followed the injudicious administration of arsenic, in no one case have I seen the constitution injured, or the health impaired, when it has been exhi-

* Hunat on the Skin, p. 17. bited in the method here advocated; on the contrary, the health has improved, the disease disappeared, and has not returned, when the arsenic was persevered in as long as was deemed necessary.

\section{Rebiens.}

Causes Génêrales des Maladies Chroniques, spécialement de la Phthisie Pulmonaire, et Moyens de Prévenir le Développement de ces Affections. Par A. Fourcault, de l'Académie de Médecine. Paris: Dusillon. 1844. pp. 480.-General Causes of Chronic Diseases, especially of Pulmonary Phthisis, and Means for Preventing the Development of these Affections.

THE sad subject of pulmonary phthisis has been treated, in different countries, by so many eminent men, and has been investigated by such a number of patient observers, both as regards its symptoms, causes, pathology, and morbid anatomy, that it hardly seemed possible that anything new on this distressing disease could be offered to the medical world. Dr. Fourcault, nothing daunted, however, enters the same field in which Sir James Clark, Louis, Laennec, Ramadge, Sir C. Scudamore, and others, have exhibited so much ingenuity. His purpose is not to introduce a new remedy for an affection which hitherto has baffled the best-directed efforts; but the author, as the title of the book indicates, offers means of preventing the development of chronic diseases in general, and of pulmonary phthisis in particular.

Dr. Fourcault (who is well known in France as a distinguished experimentalist and physiologist) asserts that the origin of tubercles is no longer problematic; and a sedentary life, inactivity, and seclusion, generate them, in most cases, by diminishing the functions of the skin, and driving back into the torrent of the circulation the materials which the cutaneous surface ought to have eliminated. (p. 71.) Alterations of the blood are thus produced, and those various kinds of cachexiæ ensue, the origin of which, says the author, was unknown before revealed by his physiological experiments. In fact, Dr. Fourcault maintains that most chronic diseases are attributable to two principal causes-the want of muscular exercise, and damp-which causes act mainly on the cutaneous surface, producing the phenomena above stated.

We are told that these facts were discovered by relying on two means of investigation - experiment and observation. The first have been highly approved of by the Academy of Sciences of Paris in 1840, the author having obtained the Montyon prize for them; the second seems trustworthy, as being an extensive comparative statistical inquiry in different countries, as France, Holland, England, and Italy. The numerical method has been in great favour of late, though it appears to us that its results should not be implicitly trusted; and we quite agree with Dr. Fourcault, when he says that a great source of error lies in the drawing up tables from postmortem examinations only, for a vast number of cases among the wealthier classes and the rural districts are thereby ex. cluded.

"Compared statistics should rest on more complete documents, referring both to the close observation of cases and to necropsies: they should include the whole population of a country, take into account the influence of trades and professions, both in large cities, manufacturing towns, and rural districts, pay due regard to topographical and meteorological peculiarities, to the usual food and habits of the inliabitants, and lastly, establish comparisons between the data thus obtained."

Repeated journeys into the above-mentioned countries, and twenty-five years' practice in several parts of his own, have given Dr. Fourcault ample opportunities to carry out his investigations, and we must say that we find in his book very valuable facts concerning the influence of sedentary habits and trades, the effect of confinement upon animals, the influence of damp, that of physical agents on the development of organs, the effects of heredity, \&c. Some are not new, but 